\title{
Comparison of Molecular Structures Determined by Electron Diffraction and Spectroscopy. Ethane and Diborane
}

\author{
Kozo Kuchisu \\ Department of Chemistry, Faculty of Science, The University of Tokyo, Bunkyo-ku, Tokyo, Japan and \\ Department of Chemistry, University of Michigan, ${ }^{*}$ Ann Arbor, Michigan 48104
}

(Received 15 July 1968)

\begin{abstract}
Gas-phase average structures for the ground-vibrational state $\left(r_{z}\right)$ for ethane and diborane have been determined by a critical comparison of the experimental results obtained from electron diffraction (average internuclear distances $r_{q}$ ) and those obtained from high-resolution infrared and Raman spectroscopy (rotational constants $\left.B_{z}^{(a)}\right)$. Experimental values have been taken from the recent literature and converted into the average structure $\left(r_{z}\right.$ or $\left.r_{\alpha}^{0}\right)$. The $r_{0}$ and $r_{\alpha}^{0}$ distances determined from electron diffraction carry uncertainties less than those in the $r_{z}$ distances determined from rotational constants, because the latter structures are very sensitive to assumptions about the unknown isotope differences in the structures. On the other hand, the average moments of inertia from spectroscopy are much more precise than those calculated from diffraction internuclear distances. Examinations of the data have led to the following $r_{\text {a }}$ structures with standard errors:
\end{abstract}

For $\mathrm{C}_{2} \mathrm{H}_{6}$,

$$
r_{z}(\mathrm{C}-\mathrm{H})=1.095_{7} \pm 0.002 \AA, \quad r_{s}(\mathrm{C}-\mathrm{C})=1.531_{9} \pm 0.002 \AA, \quad \text { and } \quad \angle \mathrm{C}-\mathrm{C}-\mathrm{H}=111.5^{\circ} \pm 0.3^{\circ} ;
$$

for $\mathrm{C}_{2} \mathrm{D}_{6}$,

$$
r_{z}(\mathrm{C}-\mathrm{D})=1.094_{1} \pm 0.002 \AA, \quad r_{3}(\mathrm{C}-\mathrm{C})=1.530_{0} \pm 0.002 \AA, \quad \text { and } \quad \angle \mathrm{C}-\mathrm{C}-\mathrm{D}=111.4^{\circ} \pm 0.3^{\circ} ;
$$

and for $\mathrm{B}_{2} \mathrm{H}_{6}$,

$$
\begin{gathered}
r_{z}\left(\mathrm{~B}-\mathrm{H}_{t}\right)=1.19_{2} \pm 0.01 \AA, \quad r_{z}\left(\mathrm{~B}-\mathrm{H}_{b}\right)=1.32_{0} \pm 0.005 \AA, \quad r_{z}(\mathrm{~B}-\mathrm{B})=1.77_{0} \pm 0.005 \AA, \\
\angle \mathrm{H}_{t}-\mathrm{B}-\mathrm{H}_{t}=121_{.8}^{\circ} \pm 3^{\circ}, \quad \text { and } \quad \angle \mathrm{H}_{b}-\mathrm{B}-\mathrm{H}_{b}=96.6^{\circ} \pm 0.5^{\circ} .
\end{gathered}
$$

It was possible to increase the resolving power of the diffraction analysis of diborane by inclusion of calculated B-H mean amplitudes.

The effective complementary use of electron-diffraction and spectroscopic data for determining reliable gas-phase structures and the relative merits of the two alternative representations of the average siructure $\left(r_{a}\right.$ and $\left.r_{x}\right)$ have been discussed.

\section{INTRODUCTION}

Previous theoretical studies ${ }^{1-5}$ have given a practical method for comparing the molecular structures determined in the gas phase by high-resolution spectroscopy and electron diffraction. The method ${ }^{1}$ makes use of the "average" moments of inertia $I_{\alpha}^{(z)}(\alpha=a, b, c)$ obtained from rotation or rotation-vibration spectroscopy and of the internuclear distances $r_{\alpha}{ }^{0}$ obtained from electron diffraction. Experimental values of $I_{\alpha}^{(z)}$ and $r_{\alpha}{ }^{0}$ can be obtained from the direct observables of spectroscopy and electron diffraction, namely, the ground-state rotational constants $A_{0}, B_{0}, C_{0}$, and the $r_{a}$ distances, respectively, by making numerical corrections for various vibrational effects. The corrections are usually so small that only an approximate knowledge of the intramolecular potential function is necessary. Except for systems

\footnotetext{
* This research was supported by a grant from the National Science Foundation.

1 Y. Morino, K. Kuchitsu, and T. Oka, J. Chem. Phys. 36, 1108 (1962).

2 T. Oka, J. Phys. Soc. Japan 15, 2274 (1960).

(a) D. R. Herschbach and V. W. Laurie, J. Chem. Phys. 37, 1668 (1962). (b) V. W. Laurie and D. R. Herschbach, J. Chem. Phys. 37, 1687 (1962).

K. Kuchitsu and L. S. Bartell, J. Chem. Phys. 36, 2460 (1962).

6 K. Kuchitsu and L. S. Bartell, J. Chem. Phys. 36, 2470 (1962).
}

with very large amplitudes of vibration, the $r_{\alpha}{ }^{0}$ distances derived in this way should be essentially equivalent to the distances between the zero-point average atomic positions represented by $I_{\alpha}^{(z)}$ (the so-called $r_{2}$ distances) to the accuracy of current experiments and conversions.

The structures of a number of simple polyatomic molecules have been studied by this method of critical comparison. Molecules investigated so far may be classified into two categories: The first group is comprised of molecules such as $\mathrm{CH}_{4},{ }^{1,3,6} \mathrm{CO}_{2},{ }^{7} \mathrm{CS}_{2},{ }^{8} \mathrm{BF}_{3},{ }^{9}$ and $\mathrm{NH}_{3},{ }^{10}$ for which the structure of any one isotope species can be determined uniquely by spectroscopy. The spectroscopic $r_{z}$ structures thus far determined have agreed satisfactorily with those determined by electron diffraction, giving experimental confirmation of the present method. The second category includes

' L. S. Bartell, K. Kuchitsu, and R. J. deNeui, J. Chem. Phys. 35,1211 (1961).

7 Y. Murata, T. Fukuyama, and M. Tanimoto, "Unit for the Precise Measurement of Electron-Diffraction Intensities by Gas Molecules. II," Bull. Chem. Soc. Japan (to be published).

${ }^{8}$ Y. Morino and T. Iijima, Bull. Chem. Soc. Japan 35, 1661 (1962).

9 K. Kuchitsu and S. Konaka, J. Chem. Phys. 45, 4342 (1966). Note that in Eq. (9) of this reference the coefficient of $\Sigma_{0^{\prime}}\left(\zeta_{\text {soos }}{ }^{(\alpha)}\right)^{2}$ should read -3 instead of +1

${ }^{10} \mathrm{~K}$. Kuchitsu, J. P. Guillory, and L. S. Bartell, J. Chem. Phys. 49,2488 (1968). 
molecules such as ethylene, ${ }^{11}$ cyanogen, ${ }^{12}$ glyoxal, ${ }^{13}$ and butadiene, ${ }^{13}$ for which a spectroscopic structure determination requires either a combination of isotope data or assumptions about the structure. Since the average structure is, in general, different from the equilibrium structure, there should be finite differences among the average structures of various isotope species because of vibrational effects, and they must be taken into account in the analysis. ${ }^{3 b}$ Structures derived for molecules in the second category may be so sensitive to assumptions about isotope effects ${ }^{14,15}$ that uncertainties from this origin can be much larger than those due to experimental errors in the average moments of inertia. In such cases, the combination of electron-diffraction and spectroscopic data may be significantly more powerful than either technique alone.

The average structures of two molecules of basic importance in structural chemistry, i.e., ethane and diborane (and their perdeuterides) have been examined in the present study. Since both of them belong to the second category, the $r_{\alpha}^{0}$ distances derived from electron diffraction have been used in combination with the average moments of inertia from spectroscopy to find their best available $r_{z}$ structures.

\section{ETHANE AND DEUTEROETHANE}

Precise experimental values of the $r_{g}$ distances from electron diffraction ${ }^{16}$ and the ground-state rotational

TABLE I. Mean amplitudes for ethane and ethane-do (in Angstrom units).

\begin{tabular}{cccc}
\hline \hline & $l_{\Omega}(\mathrm{obs}) \mathrm{a}$ & $\left\langle\Delta z^{2}\right\rangle_{\mathrm{T}}^{1 / 2 \mathrm{~b}}$ & $\left\langle\Delta z^{2}\right\rangle_{0}{ }^{1 / 20}$ \\
\hline $\mathrm{C}_{2} \mathrm{H}_{6} \mathrm{C}-\mathrm{H}$ & $0.076_{0} \pm 0.001_{0}$ & $0.078_{5}$ & $0.078_{6}$ \\
$\mathrm{C}-\mathrm{C}$ & $0.049_{6} \pm 0.001_{0}$ & $0.050_{9}$ & $0.050_{6}$ \\
$\mathrm{C} \cdots \mathrm{H}$ & $0.106_{7} \pm 0.001_{6}$ & $0.108_{8}$ & $0.108_{2}$ \\
$\mathrm{C}_{2} \mathrm{D}_{6} \mathrm{C}-\mathrm{D}$ & $0.066_{8} \pm 0.001_{0}$ & $0.067_{0}$ & $0.067_{0}$ \\
$\mathrm{C}-\mathrm{C}$ & $0.051_{7} \pm 0.001_{6}$ & $0.050_{8}$ & $0.050_{4}$ \\
$\mathrm{C} \cdots \mathrm{D}$ & $0.093_{1} \pm 0.001_{6}$ & $0.095_{0}$ & $0.093_{2}$ \\
\hline
\end{tabular}

Reference 16. Uncertainties indicate standard deviations.

b Calculated for room temperature.

- Calculated for $0^{\circ} \mathrm{K}$.

${ }^{11}$ K. Kuchitsu, J. Chem. Phys. 44, 906 (1966).

${ }^{12} \mathbf{Y}$. Morino, K. Kuchitsu, Y. Hori, and M. Tanimoto, Bull. Chem. Soc. Japan 41, 2349 (1968).

${ }^{13} \mathrm{~K}$. Kuchitsu, T. Fukuyama, and Y. Morino, J. Mol. Struct. 1, 463 (1968).

${ }_{14} \mathrm{~K}$. Kivelson, E. B. Wilson, Jr., and D. R. Lide, Jr., J. Chem. Phys. 32, 205 (1960).

${ }_{15}$ W. J. Lafferty, D. R. Lide, and R. A. Toth, J. Chem. Phys. 43, 2063 (1965).

${ }_{16}$ L. S. Bartell and H. K. Higginbotham, J. Chem. Phys, 42, 851 (1965).
TABLE II. Rotational constants for ethane and ethane-d $d_{6}$ (in $\mathrm{cm}^{-1}$ units).

\begin{tabular}{ccc}
\hline \multicolumn{1}{c}{$A_{0}$} & \multicolumn{1}{c}{$B_{0}$} \\
$\mathrm{C}_{2} \mathrm{H}_{8}$ & $2.671 \pm 0.005^{\mathrm{b}}$ & $0.66310 \pm 0.00007^{\mathrm{d}}$ \\
& & $0.66313_{5} \pm 0.00002_{4}^{\circ}$ \\
$\mathrm{C}_{2} \mathrm{D}_{6}$ & $1.3416 \pm 0.0009^{\circ}$ & $0.45973 \pm 0.00008^{\mathrm{f}}$ \\
& $A_{z^{\mathrm{a}}}$ & $B_{2}^{\mathrm{a}}$ \\
$\mathrm{C}_{2} \mathrm{H}_{6}$ & 2.682 & $0.6621_{6}$ \\
$\mathrm{C}_{2} \mathrm{D}_{3}$ & 1.345 & $0.4592_{2}$ \\
\hline
\end{tabular}

average rotational constants calculated in the present study from the corresponding experimental rotational constants $A_{\bullet}$ and $B_{0}$.

b Reference 18.

- Reference 17.

d Reference 20 .

Reference 21.

${ }^{f}$ References 19 and 26.

constants $A_{0}$ and $B_{0}$ from $\operatorname{Raman}^{17,18}$ and infrared ${ }^{19-21}$ spectroscopy have been reported for $\mathrm{C}_{2} \mathrm{H}_{6}$ and $\mathrm{C}_{2} \mathrm{D}_{6}$. They were converted into the $r_{\alpha}{ }^{0}$ and the $A_{z}, B_{z}$ constants $^{1}$ by means of the mean-square amplitudes and corrections for vibration-rotation interactions (Tables I and II). The conversions were based on the following equations $\mathrm{s}^{9,11}$

$$
\begin{aligned}
r_{\alpha}^{0} & =\left[\lim \left(T \rightarrow 0^{\circ} \mathrm{K}\right) r_{g}\right]-K_{0}-\delta r_{\mathrm{cent}} \\
& \simeq r_{g}-\frac{3}{2} a\left(\left\langle\Delta z^{2}\right\rangle_{T}-\left\langle\Delta z^{2}\right\rangle_{0}{ }^{\circ} \mathrm{K}\right)-K_{0}-\delta r_{\text {cent }},
\end{aligned}
$$

where

and

$$
K_{0}=\left(\left\langle\Delta x^{2}\right\rangle_{0}{ }^{\circ} \mathrm{K}+\left\langle\Delta y^{2}\right\rangle_{0}{ }^{\circ} \mathrm{K}\right) / 2 r
$$

$$
\begin{aligned}
& B_{z}{ }^{(\alpha)}=h / 8 \pi^{2} c I_{\alpha}{ }^{(z)}
\end{aligned}
$$

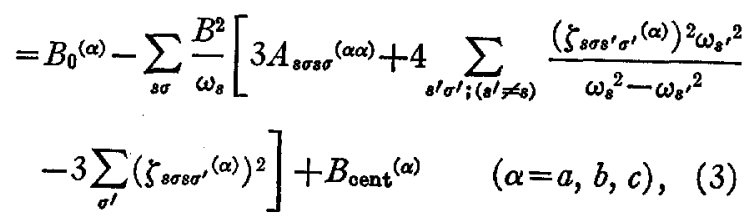

and on the force constants ${ }^{22}$ which reproduce the observed values ${ }^{17,18,23}$ of fundamental frequencies and degenerate Coriolis constants satisfactorily. In Eqs. (1) and (2) $x, y$, and $z$ are a set of local Cartesian displacement coordinates for a particular atom pair with $z$

${ }^{17}$ D. W. Lepard, D. M. C. Sweeny, and H. L. Welsh, Can. J. Phys. 40, 1567 (1962)

${ }_{18}$ D. W. Lepard, D. E. Shaw, and H. L. Welsh, Can. J. Phys. 44, 2353 (1966).

${ }^{19} \mathrm{H}$. C. Allen, Jr., and E. K. Plyler, J. Chem. Phys. 31, 1062 (1959).

${ }_{20}$ W. J. Lafferty and E. K. Plyler, J. Chem. Phys. 37, 2688 (1962)

${ }_{21}$ A. R. H. Cole, W. J. Lafferty, and R. J. Thibault, "Rotational Fine Structure of the Perpendicular Band, $\nu_{7}$, of Ethane," J. Mol. Spectry. (to be published).

${ }_{22}$ I. Nakagawa (private communication).

${ }^{23}$ G. E. Hansen and D. M. Dennison, J. Chem. Phys. 20, 313 (1952). 
TABLE III. Average internuclear distances for ethane and ethane- $d_{6}$ (in Ångstrom units).

\begin{tabular}{cccc}
\hline & $r_{o}(\mathrm{obs})$ & $K_{0}{ }^{\mathrm{b}}$ & $r_{\alpha}^{0 \mathrm{e}}$ \\
\hline $\mathrm{C}_{2} \mathrm{H}_{6} \mathrm{C}-\mathrm{H}$ & $1.112_{2} \pm 0.001_{2}$ & $0.015_{7}$ & $1.097_{1} \pm 0.002_{0}$ \\
$\mathrm{C}-\mathrm{C}$ & $1.534_{0} \pm 0.001_{1}$ & 0.0006 & $1.533_{3} \pm 0.001_{5}$ \\
$\mathrm{C} \cdots \mathrm{H}$ & $2.194_{1} \pm 0.001_{9}$ & $0.006_{2}$ & $2.187_{5} \pm 0.002_{5}$ \\
$\mathrm{C}_{2} \mathrm{D}_{6} \mathrm{C}-\mathrm{D}$ & $1.107_{1} \pm 0.001_{2}$ & $0.011_{2}$ & $1.095_{9} \pm 0.002_{0}$ \\
$\mathrm{C}-\mathrm{C}$ & $1.532_{3} \pm 0.001_{1}$ & 0.0009 & $1.531_{3} \pm 0.001_{5}$ \\
$\mathrm{C} \cdots \mathrm{D}$ & $2.189_{2} \pm 0.002_{0}$ & $0.004_{4}$ & $2.183_{8} \pm 0.002_{5}$ \\
\hline
\end{tabular}

a Observed values by electron diffraction. ${ }^{16}$ Uncertainties indicate stand. ard deviatlons.

b Calculated by the use of force constants ${ }^{22}$ by Eq. (2),

- Calculated from ${ }_{g}$ by Eq. (1).

taken in the direction of the equilibrium positions of atoms. Trivial effects of centrifugal distortion ${ }^{24,25}$ were ignored. The differences between $r_{a}$ and $r_{a}{ }^{0}$ distances (Table III) and those between $A_{0}, B_{0}$, and $A_{z}, B_{z}$ constants (Table II) exceed the corresponding experimental errors by an order of magnitude and, hence, the above corrections for the vibrational effect are found to be significant. The mean amplitudes $l_{g}$ observed by Bartell and Higginbotham ${ }^{16}$ agree well with the present results of calculations (Table I).

In order to determine the structure from spectroscopic data alone, one has to make at least two assumptions about the structures since there are three independent parameters for each isotope species but only two observable rotational constants for each. Shaw et $a l .{ }^{26}$ determined effective $r_{0}$ structures on the assumptions that the $\mathrm{H}-\mathrm{C}-\mathrm{H}$ and $\mathrm{D}-\mathrm{C}-\mathrm{D}$ angles were identical and that the $\mathrm{C}-\mathrm{C}$ distances in $\mathrm{C}_{2} \mathrm{H}_{6}$ and $\mathrm{C}_{2} \mathrm{D}_{6}$ were also identical. In the present analysis, the following assumptions were made in regard to the isotope differences:

$$
r_{2}(\mathrm{C}-\mathrm{H})-r_{z}(\mathrm{C}-\mathrm{D})=0.002 \pm 0.002 \AA,
$$

and

$$
r_{z}\left(\mathrm{C}-\mathrm{C}, \mathrm{C}_{2} \mathrm{H}_{6}\right)-\boldsymbol{r}_{z}\left(\mathrm{C}-\mathrm{C}, \mathrm{C}_{2} \mathrm{D}_{6}\right)=0 \pm 0.002 \AA .
$$

The above estimates were based on the relation ${ }^{1,11}$

$$
\begin{aligned}
r_{z}(\mathrm{H}) & -r_{z}(\mathrm{D}) \\
\quad & \simeq\left[r_{g}(\mathrm{H})-r_{g}(\mathrm{D})\right]_{0}{ }^{\circ} \mathrm{K}-\left[K_{0}(\mathrm{H})-K_{0}(\mathrm{D})\right] \\
& \simeq \frac{3}{2} a\left[\left\langle\Delta z^{2}\right\rangle(\mathrm{H})-\left\langle\Delta z^{2}\right\rangle(\mathrm{D})\right]_{0}{ }^{\circ} \mathrm{K}-\left[K_{0}(\mathrm{H})-K_{0}(\mathrm{D})\right],
\end{aligned}
$$

with the numerical values listed in Tables I and III. The parameters of bond-stretching anharmonicity, $a_{\mathrm{C}-\mathrm{H}}$ and $a_{\mathrm{C}-\mathrm{C}}$, were assumed to be $2.6 \pm 0.5 \AA^{-1}$ and $2.0 \pm$ $0.5 \AA^{-1}$, respectively, and allowance was made for

${ }^{24}$ M. Iwasaki and K. Hedberg, J. Chem. Phys. 36, 2961 (1962).

${ }^{25} \mathrm{~T}$. Oka and Y. Morino, J. Mol. Spectry. 6, 472 (1961).

${ }^{26}$ D. E. Shaw, D. W. Lepard, and H. L. Welsh, J. Chem. Phys. 42, 3736 (1965). possible additional uncertainties. ${ }^{27}$ No basis exists for estimating the difference between the average angles $\mathrm{H}-\mathrm{C}-\mathrm{H}$ and $\mathrm{D}-\mathrm{C}-\mathrm{D}$ with more certainty.

The corresponding $r_{z}$ structure for $\mathrm{C}_{2} \mathrm{H}_{6}$ is found to be $\quad r_{z}(\mathrm{C}-\mathrm{H})=1.093 \pm 0.008 \quad \AA, \quad r_{z}(\mathrm{C}-\mathrm{C})=1.534 \mp$ $0.012 \AA$, and $\alpha_{z}(\mathrm{H}-\mathrm{C}-\mathrm{H})=107.5^{\circ} \pm 1.0^{\circ}$. The resulting isotope difference between the $\mathrm{HCH}$ and DCD angles, which depends essentially on the assumption of $r_{z}(\mathrm{C}-\mathrm{H})-r_{z}(\mathrm{C}-\mathrm{D})$ alone, turns out to be $6^{\prime} \pm 16^{\prime}$.

As pointed out by Laurie and Herschbach ${ }^{3 b}$ and for ClCN by Lafferty, Lide, and Toth, ${ }^{15}$ the average structure is quite sensitive to the assumptions about isotope differences. The ambiguity in the structure introduced from this origin is several fold larger than that propagated from the uncertainties in the experimental rotational constants listed in Table II, even when the error in the 0 to $z$ conversion is included in the latter uncertainties. Therefore, it seems difficult to determine the $r_{z}$ structure with less ambiguity from rotational constants alone, no matter how accurate they may be, unless a better a priori estimate can be made for the differences in isotope structures.

On the other hand, unique sets of $r_{\alpha}{ }^{0}$ distances have been determined from electron diffraction alone separately for $\mathrm{C}_{2} \mathrm{H}_{6}$ and $\mathrm{C}_{2} \mathrm{D}_{6}$. As shown in Table IV, the average moments of inertia calculated from those $r_{\alpha}{ }^{0}$ distances are only about $0.2 \%$ larger than their spectroscopic counterparts, which are an order of magnitude

\begin{tabular}{|c|c|c|}
\hline & $I_{a}^{(2)}$ & $I_{b}^{(z)}$ \\
\hline${ }^{\mathrm{C}_{2} \mathrm{H}_{6}} \underset{\mathrm{ED}^{\mathrm{SP}}(\mathrm{calc})^{\mathrm{b}}}{ }$ & $\begin{array}{l}6.28_{6} \pm 0.01_{2} \\
6.30_{6} \pm 0.02_{5}\end{array}$ & $\begin{array}{l}25.459 \pm 0.003 \\
25.50_{7} \pm 0.03_{4}\end{array}$ \\
\hline $\begin{array}{rl}\mathrm{C}_{2} \mathrm{D}_{6} & \mathrm{SP} \text { (obs) } \\
\text { ED (calc) }\end{array}$ & $\begin{array}{l}12.534 \pm 0.008 \\
12.58_{6} \pm 0.05_{0}\end{array}$ & $\begin{array}{l}36.709 \pm 0.006 \\
36.76_{4} \pm 0.06_{7}\end{array}$ \\
\hline
\end{tabular}
more precise..$^{28}$ The nearly uniform discrepancies observed, although none of them are really significant,

Table IV. Average moments of inertia for ethane and ethane- $d_{6}$ (atomic mass units $\times$ square Ångstrom units).

a From the observed average rotational constants $A$, and $B$, given in Table II.

$\mathrm{b}$ Calculated from the $r_{\alpha}^{0}$ distances by electron diffraction given in Table I.

${ }^{27}$ From this relation, a similar $r_{z}(\mathrm{C}-\mathrm{H})-r_{z}(\mathrm{C}-\mathrm{D})$ may be expected for ethylene. Hence, the assumption made in Ref. 11 for the difference, $0.004_{0} \AA$, transferred from triatomic molecules and tetratomic molecules, may have been an overestimate. An alternative assumption, $0.002 \AA$, will shift the spectroscopic $r$ structures of ethylene ( $r_{\mathrm{CC}}, r_{\mathrm{CH}}$, and $\alpha_{\mathrm{CCH}}$ listed in Table VII of Ref. 11) by $+0.0020 \AA,-0.0026 \AA$, and $+10^{\prime}$, respectively, and accordingly, the most probable $r_{z}$ structure will be: $r(\mathrm{C}-\mathrm{H})=$ $1.089 \pm 0.003 \AA, \quad r(\mathrm{C}-\mathrm{C})=1.336 \pm 0.003 \AA$, and $\angle \mathrm{C}-\mathrm{C}-\mathrm{H}=$ $121.9^{\circ} \pm 0.4^{\circ}$.

${ }_{28}$ The rotational constants $A_{0}$ for $\mathrm{C}_{2} \mathrm{H}_{6}$ reported earlier (a) $2.589 \mathrm{~cm}^{-1}$ [L. G. Smith, J, Chem. Phys. 17, 139 (1949)] and (b) $2.578 \mathrm{~cm}^{-1}$ [J. Ramanko, T. Feldman, and H. L. Weish, Can. J. Phys. 33, 588 (1955) ] lead to $I_{a}^{(s)}$ of 6.484 and 6.511 amu. $\AA^{2}$, respectively. Since they appear to deviate significantly from the present estimate from electron diffraction, $6.30_{5} \pm 0.02_{5}$ amu $\AA^{2}$, the $A_{0}$ constant revised by Lepard et al. ${ }^{18}(2.671 \pm$ $\left.0.005 \mathrm{~cm}^{-1}, I_{a}^{(z)}=6.286 \pm 0.012 \mathrm{amu} \cdot \AA^{2}\right)$ seems to be more reasonable. 
suggest that a slight systematic error (about $0.09 \%$ for $\mathrm{C}_{2} \mathrm{H}_{6}$ and about $0.07 \%$ for $\mathrm{C}_{2} \mathrm{D}_{6}$ ) may be present in the scale-factor measurement of electron diffraction..$^{16}$ Accordingly, a tentative adjustment within the limit of experimental errors of the $r_{\alpha}^{0}$ distances was made primarily on this basis, so as to reproduce the spectroscopic moments of inertia exactly. The $r_{g}$ and $r_{z}$ distances shown in Table $V$ has been obtained in this way. The $r_{\theta}(\mathrm{C}-\mathrm{H})$ and $r_{0}(\mathrm{C}-\mathrm{D})$ bond distances in $\mathrm{C}_{2} \mathrm{H}_{6}$ and $\mathrm{C}_{2} \mathrm{D}_{6}$ appear to be longer than the corresponding distances ${ }^{6}$ in $\mathrm{CH}_{4}$ and $\mathrm{CD}_{4}$ by about $0.004 \AA$, whereas the projected distances $r_{z}$ seem to be in the opposite direction; the difference $r_{g}-r_{z}$ is much larger for $\mathrm{C}_{2} \mathrm{H}_{6}\left(\mathrm{C}_{2} \mathrm{D}_{6}\right)$ than for $\mathrm{CH}_{4}\left(\mathrm{CD}_{4}\right)$, because the amplitudes of the $\mathrm{H}(\mathrm{D})$ atoms perpendicular to the $\mathrm{C}-\mathrm{H}(\mathrm{C}-\mathrm{D})$ bond are much larger in $\mathrm{C}_{2} \mathrm{H}_{6}\left(\mathrm{C}_{2} \mathrm{D}_{6}\right)$ than in $\mathrm{CH}_{4}\left(\mathrm{CD}_{4}\right){ }^{5}$

The effective $r_{0}$ structure reported by Shaw et al..$^{26}$ is very close to the present $r_{z}$ structure. The difference of $0.001_{9} \pm 0.002 \AA$ in the $r_{z}(\mathrm{C}-\mathrm{C})$ distances for $\mathrm{C}_{2} \mathrm{H}_{6}$ and $\mathrm{C}_{2} \mathrm{D}_{6}$ (the $r_{z}$ secondary-isotope effect ${ }^{16,29}$ ) is similar to the $r_{a}$ secondary isotope effect, $0.0016 \pm 0.0007 \AA$, reported by Bartell and Higginbotham. ${ }^{16}$ However, it has not been possible to decrease the uncertainty by the additional account of spectroscopic rotational constants. A more definitive solution of this problem depends crucially either on the experimental accuracy of electron diffraction or on the correct estimation of the $r_{z}$ primary isotope effects on the $\mathrm{C}-\mathrm{H}$ distance and the $\mathrm{H}-\mathrm{C}-\mathrm{H}$ angle.

\section{DIBORANE}

A set of $F$ matrix elements was obtained from observed vibrational frequencies ${ }^{30}$ by a normal-coordinate analysis, ${ }^{31}$ and used in the calculation of mean-square

Table V. Average structures for ethane and ethane- $d_{6}^{\mathrm{s}}$ (distances in Ångstrom units).

\begin{tabular}{ccccc}
\hline & $r_{\sigma}$ & $r_{z}$ & \multicolumn{1}{c}{$\sigma^{\mathrm{b}}$} & $r_{0^{\circ}}$ \\
\hline $\mathrm{C}_{2} \mathrm{H}_{6} \mathrm{C}-\mathrm{H}$ & $1.110_{8}$ & $1.095_{7}$ & 0.002 & $1.095 \pm 0.002$ \\
$\mathrm{C}-\mathrm{C}$ & $1.532_{6}$ & $1.531_{9}$ & 0.002 & $1.534 \pm 0.002$ \\
$\mathrm{C} \cdots \mathrm{H}$ & $2.192_{1}$ & $2.185_{5}$ & 0.003 & $(2.188 \pm 0.004)$ \\
$\angle \mathrm{C}-\mathrm{C}-\mathrm{H}$ & $\cdots$ & $111.5^{\circ}$ & $0.3^{\circ}$ & $\left(111.6^{\circ} \pm 0.2^{\circ}\right)$ \\
$\angle \mathrm{H}-\mathrm{C}-\mathrm{H}$ & $\cdots$ & $107.4^{\circ}$ & $0.3^{\circ}$ & $107.8^{\circ} \pm 0.2^{\circ}$ \\
$\mathrm{C}_{2} \mathrm{D}_{6} \mathrm{C}-\mathrm{D}$ & $1.105_{8}$ & $1.094_{1}$ & 0.002 & $1.093 \pm 0.001$ \\
$\mathrm{C}-\mathrm{C}$ & $1.531_{0}$ & $1.530_{0}$ & 0.002 & $(1.534)$ \\
$\mathrm{C} \cdots \mathrm{D}$ & $2.187_{5}$ & $2.182_{1}$ & 0.003 & $(2.187)$ \\
$\angle \mathrm{C}-\mathrm{C}-\mathrm{D}$ & $\cdots$ & $111.4^{\circ}$ & $0.3^{\circ}$ & $\left(111.6^{\circ}\right)$ \\
$\angle \mathrm{D}-\mathrm{C}-\mathrm{D}$ & $\cdots$ & $107.4^{\circ}$ & $0.3^{\circ}$ & $\left(107.8^{\circ}\right)$ \\
\hline \hline
\end{tabular}

- See Ref. 16, Table IV for the comparison of ethane structures studied earlier.

b Estimated standard errors for $r_{g}$ and $r_{z}$ parameters.

- Effective ro structure determined by Shaw et al.2a

L. S. Bartell, J. Chem. Phys. 36, 3495 (1962).

${ }^{30}$ R. C. Lord and E. Nielsen, J. Chem. Phys. 19, 1 (1951). T. Ogawa and T. Miyazawa, Spectrochim. Acta 20, 557 (1964).

11 The author is grateful to I. Nakagawa for providing his unpublished calculations on the normal-coordinate analysis of diborane and for his helpful discussions.
TABLe VI. Mean amplitudes for diborane and diborane- $d_{\sigma}$ (in Angstrom units).

\begin{tabular}{|c|c|c|c|c|}
\hline & & $l_{g}(\mathrm{obs})^{\mathrm{a}}$ & $\left\langle\Delta z^{2}\right\rangle T^{1 / 2}$ & $\left\langle\Delta z^{2}\right\rangle_{0}^{1 / 2}$ \\
\hline \multirow[t]{4}{*}{$\mathrm{B}_{2} \mathrm{H}_{6}$} & $\mathrm{~B}-\mathrm{H}_{t}$ & $0.073_{4} \pm 0.006$ & $0.084_{5}$ & $0.084_{5}$ \\
\hline & $\mathrm{B}-\mathrm{H}_{b}$ & $0.085_{7} \pm 0.009$ & $0.101_{6}$ & $0.101_{5}$ \\
\hline & B-B & $0.060_{8} \pm 0.002$ & $0.061_{1}$ & $0.059_{9}$ \\
\hline & $\mathrm{B} \cdot \cdots \mathrm{H}_{t}$ & $0.117_{8} \pm 0.005$ & $0.129_{1}$ & $0.122_{7}$ \\
\hline \multirow[t]{4}{*}{$\mathrm{B}_{2} \mathrm{D}_{6}$} & $\mathrm{~B}-\mathrm{D}_{t}$ & $0.064_{3} \pm 0.005$ & $0.072_{3}$ & $0.072_{3}$ \\
\hline & $\mathrm{B}-\mathrm{D}_{b}$ & $0.075_{6} \pm 0.006$ & $0.087_{\mathrm{L}}$ & $0.086_{8}$ \\
\hline & B-B & $0.059_{5} \pm 0.002$ & $0.060_{8}$ & $0.059_{4}$ \\
\hline & $\mathrm{B} \cdots \mathrm{D}_{t}$ & $0.104_{9} \pm 0.005$ & $0.117_{7}$ & $0.106_{4}$ \\
\hline
\end{tabular}

\& Electron diffraction analysis." The diffraction values can be brought into conformity with the present calculated values by decreasing the differences between bridge and terminal bond lengths, as shown in Fig. 6 of Ref. 32.

amplitudes and Coriolis coupling constants with a HITAC 5020E computer of the Computer Center, University of Tokyo. Parallel amplitudes are compared in Table VI with those observed by Bartell and Carroll. ${ }^{32}$ Corresponding to Tables II and III for ethane, Tables VII and VIII list average rotational constants and $r_{\alpha}{ }^{0}$ distances.

As pointed out by Bartell, ${ }^{33}$ secondary isotope effects on $\mathrm{C}-\mathrm{C}$ and $\mathrm{B}-\mathrm{B}$ amplitudes for ethane (Table I) and diborane (Table VI), respectively, are very small in contrast to the significant primary isotope effects on $\mathrm{C}-\mathrm{H}$ and $\mathrm{B}-\mathrm{H}$ amplitudes. The amplitudes of bridge $\mathrm{B}-\mathrm{H}$ bonds are about $20 \%$ larger than those of terminal B-H bonds, in accordance with the observation and discussion in Ref. 32 in terms of Badger's empirical rule. Nevertheless, the calculated B-H and B-D amplitudes are slightly but consistently larger than the corresponding rather approximate experimental values. According to Fig. 6 of this reference, the B-H (B-D) amplitudes correlate strongly with the difference between the bridge and terminal $\mathrm{B}-\mathrm{H}$ distances, greatly reducing experimental accuracy, and probably the present systematic discrepancies originate from this difficulty. For this reason, the additional information provided by the calculated amplitudes can be used to augment materially the accuracy of the $\mathrm{B}-\mathrm{H}$ distances derived from the diffraction intensities. The calculated amplitudes, together with Figs. 5 and 6 of Ref. 32, lead to the corrected sets of $r_{g}$ values $\left[r_{g}(\right.$ cor $\left.)\right]$ shown in Table VIII.

Average rotational constants for the ${ }^{10} \mathrm{~B}_{2} \mathrm{H}_{6}$ and ${ }^{11} \mathrm{~B}_{2} \mathrm{H}_{6}$ species have been derived from Lafferty's experimental constants, ${ }^{34}$ which are so precise that the 0 to $z$ corrections are not trivial. Since structural parameters

\footnotetext{
${ }^{22}$ L. S. Bartell and B. L. Carroll, J. Chem. Phys. 42, 1135 (1965). For structure of diborane, see Fig. 4 of this reference.

${ }^{3}$ L. S. Bartell, J. Chem. Phys. 42, 1681 (1965).

${ }^{34}$ W. J. Lafferty (private communication, December 1967), to which the author is indebted. T. Coyle, W. J. Lafferty, and A. G. Maki, J. Mol. Spectry. (to be:published).
} 
TABLE VII. Rotational constants for diborane (in $\mathrm{cm}^{-1}$ units).

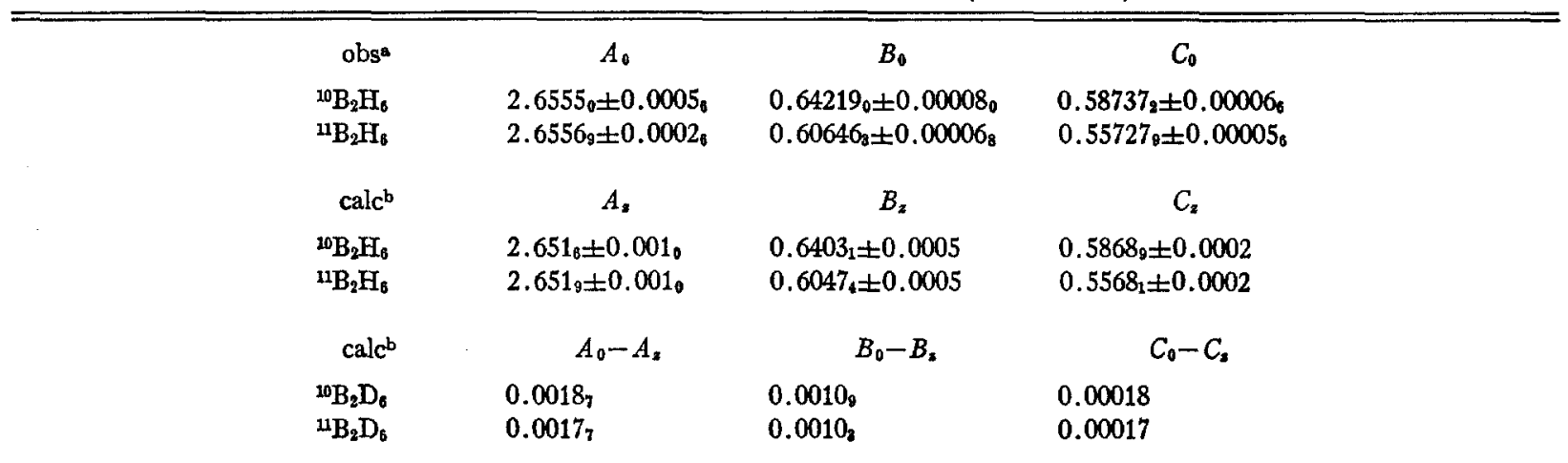

abserved values by infrared spectroscopy with twice standard devia-

b Average rotational constants and correction terms, respectively, calcu tions. ${ }^{34}$ lated in the present study.

TABLE VIII. Average internuclear distances for diborane and diborane-d (in Ångstrom units).

\begin{tabular}{|c|c|c|c|c|}
\hline & $r_{0}(\mathrm{obs}) \mathrm{a}$ & $r_{o}(\operatorname{corr})^{b}$ & $K_{0}$ & $r_{\alpha}^{0}$ \\
\hline $\mathrm{B}_{2} \mathrm{H}_{6} \mathrm{~B}-\mathrm{H}_{\boldsymbol{t}}$ & $1.196_{0}(+0.008,-0.006)$ & 1.208 & 0.0177 & 1.180 \\
\hline $\mathrm{B}-\mathrm{H}_{b}$ & $1.339_{3}(+0.002,-0.006)$ & 1.329 & 0.0111 & 1.329 \\
\hline $\mathrm{B}-\mathrm{B}$ & $1.775_{0} \pm 0.003_{5}$ & 1.775 & 0.0006 & 1.774 \\
\hline $\mathrm{B} \cdots \mathrm{H}_{t}$ & $2.588_{1} \pm 0.009$ & 2.588 & 0.0058 & 2.583 \\
\hline $\mathrm{B}_{2} \mathrm{D}_{6} \mathrm{~B}-\mathrm{D}_{\mathrm{t}}$ & $1.198_{0}(+0.006,-0.005)$ & 1.210 & 0.0113 & 1.187 \\
\hline $\mathrm{B}-\mathrm{D}_{b}$ & $1.333_{5}(+0.002,-0.004)$ & 1.323 & 0.0076 & 1.326 \\
\hline $\mathbf{B}-\mathbf{B}$ & $1.771_{2} \pm 0.003_{5}$ & 1.771 & 0.0011 & 1.770 \\
\hline $\mathrm{B} \cdots \mathrm{D}_{t}$ & $2.572_{3} \pm 0.008$ & 2.572 & 0.0036 & 2.569 \\
\hline
\end{tabular}

a Observed values by electron diffraction. ${ }^{32}$ Uncertainties indicate standard deviations.

b Corrected to be consistent with calculated mean amplitudes. See Figs. 5 and 6, Ref. 32.

TABLE IX. Average structures for diborane (distances in Ångstrom units).

\begin{tabular}{|c|c|c|c|c|c|}
\hline & $r_{2}(\mathrm{SP})$ & $\left(\begin{array}{c}\boldsymbol{\gamma}_{\boldsymbol{\alpha}}^{0} \\
(\mathrm{ED})\end{array}\right.$ & $\left(\mathrm{ED}^{r_{\mathrm{a}}^{0}}, \mathrm{corr}\right)$ & $r_{*}^{a}$ & $r_{0}^{b}$ \\
\hline $\mathrm{B}-\mathrm{H}_{\boldsymbol{t}}$ & $1.19_{9} \mp 0.01_{4}$ & $1.18_{0} \mp 0.009$ & $1.19_{2}$ & $1.19_{2} \pm 0.01$ & $1.200_{6} \pm 0.0018$ \\
\hline $\mathrm{B}-\mathrm{H}_{b}$ & $1.327 \pm 0.005$ & $1.329 \pm 0.008$ & $1.31_{9}$ & $1.32 \mathrm{~g} \pm 0.005$ & $1.320_{4} \pm 0.0005$ \\
\hline B-B & $1.76_{4} \pm 0.01_{4}$ & $1.774 \pm 0.004$ & 1.774 & $1.77_{0} \pm 0.005$ & $1.762_{8} \pm 0.0013$ \\
\hline$\angle \mathrm{H}_{t} \mathrm{BH}_{t}$ & $120.7^{\circ} \pm 2.4^{\circ}$ & $118.8^{\circ} \pm 3.4^{\circ}$ & $120.3^{\circ}$ & $121.8^{\circ} \pm 3^{\circ}$ & $121.0^{\circ} \pm 0.3^{\circ}$ \\
\hline$\angle \mathrm{H}_{b} \mathrm{BH}_{b}$ & $96.7^{\circ} \mp 0.5^{\circ}$ & $96.2^{\circ} \pm 0.8^{\circ}$ & $95.5^{\circ}$ & $96.5^{\circ} \pm 0.5^{\circ}$ & $96.2^{\circ} \pm 0.1^{\circ}$ \\
\hline
\end{tabular}

Estimated from infrared (SP) and electron diffraction (ED) structures. Uncertainties represent standard deviations.

$\mathrm{b}$ Effective $r_{0}$ structure $\left(r_{g}\right.$ for $\left.\mathrm{B}-\mathrm{B}\right)$ determined by Lafferty.

TABLE X. Average moments of inertia for diborane (in atomic mass units $\times$ square Angstrom units).

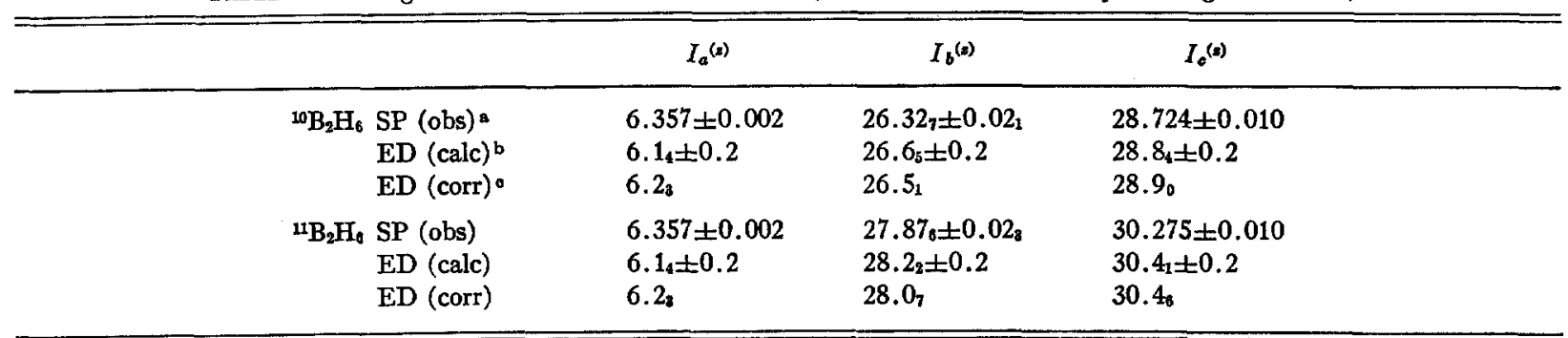


are related to the moments of inertia as

$$
\begin{aligned}
\Delta_{a} & =I_{b}^{(z)}+I_{c}^{(z)}-I_{a}^{(z)} \\
& =m_{\mathrm{B}} R^{2}+2 m_{\mathrm{H}}\left(R+2 r_{t} \cos \alpha_{t}\right)^{2}, \\
\Delta_{b} & =I_{c}^{(z)}+I_{a}^{(z)}-I_{b}^{(z)} \\
& =8 m_{\mathrm{H}} r_{t}^{2} \sin ^{2} \alpha_{t},
\end{aligned}
$$

and

$$
\begin{aligned}
\Delta_{c} & =I_{a}{ }^{(z)}+I_{b}{ }^{(z)}-I_{c}{ }^{(z)} \\
& =4 m_{\mathrm{H}} \gamma_{b}{ }^{2} \sin ^{2} \alpha_{b},
\end{aligned}
$$

where

$$
\begin{array}{cl}
r_{t}=r_{z}\left(\mathrm{~B}-\mathrm{H}_{t}\right), & r_{b}=r_{z}\left(\mathrm{~B}-\mathrm{H}_{b}\right), \\
2 \alpha_{t}=\angle \mathrm{H}_{t}-\mathrm{B}-\mathrm{H}_{t}, & 2 \alpha_{b}=\angle \mathrm{H}_{b}-\mathrm{B}-\mathrm{H}_{b},
\end{array}
$$

and

$$
R=r_{z}(\mathrm{~B}-\mathrm{B})=2 r_{b} \cos \alpha_{b},
$$

it is possible to determine all the parameters uniquely by ${ }^{10} \mathrm{~B}-{ }^{11} \mathrm{~B}$ substitution, provided some assumption is made about the isotope effect on structure. By a procedure analogous to the ethane analysis, the isotope effects were estimated from Eq. (5) to be

and

$$
r_{z}\left({ }^{10} \mathrm{~B}-{ }^{10} \mathrm{~B}\right)-r_{z}\left({ }^{11} \mathrm{~B}-{ }^{11} \mathrm{~B}\right)=0 \pm 0.001 \AA \text {, }
$$

$$
r_{z}\left({ }^{10} \mathrm{~B}-\mathrm{H}\right)-r_{z}\left({ }^{11} \mathrm{~B}-\mathrm{H}\right)=0 \pm 0.0001 \AA .
$$

The uncertainty in the $\mathrm{B}-\mathrm{B}$ isotope difference turned out to be the principal source of error in the spectroscopic $r_{z}$ structure derived in this way $\left[r_{z}(\mathrm{SP})\right.$ in Table IX]; should this uncertainty be disregarded, the $\mathrm{B}-\mathrm{B}$ and $\mathrm{B}-\mathrm{H}$ bond distances would have errors of only about $0.002 \AA$, which originate from those in the average rotational constants. On the other hand, average moments of inertia calculated from the diffraction distances (Table $\mathrm{X}$ ) have uncertainties several fold larger than those from spectroscopy because of the lack of accuracy in the diffraction parameters related to hydrogen positions.

Within the estimated uncertainties of about $0.01 \AA$, the average structures obtained from diffraction and spectroscopy are compatible. Small discrepancies between the diffraction and spectroscopic distances can be decreased if one assumes that the $r_{z}\left({ }^{10} \mathrm{~B}-{ }^{10} \mathrm{~B}\right)$ distance is larger than the $r_{x}\left({ }^{11} \mathrm{~B}-{ }^{11} \mathrm{~B}\right)$ by about $0.0005 \AA$. This suggests that the effective $a_{\mathrm{B}-\mathrm{B}}$ parameter in Eq. (5) is positive (as for any bonded atom pair) and of the order of $a_{\mathrm{C}-\mathrm{C}}\left(\sim 2 \AA^{-1}\right)$.

The estimated average structure, which is consistent with the moments of inertia and with the diffraction distances, is compared in Table IX with the effective $r_{0}$ structure $^{34}$ obtained from ground-state rotational constants without regard to the isotope difference in structure. In order to determine the average structure of diborane with more accuracy [particularly the secondary isotope difference ${ }^{32}$ between the $r_{z}(\mathrm{~B}-\mathrm{B})$ distances for $\mathrm{B}_{2} \mathrm{H}_{6}$ and $\mathrm{B}_{2} \mathrm{D}_{6}$ ], it would seem to be helpful, as in the case of ethane, to re-analyze electron diffraction intensities measured with the improved accuracy now available, taking into account the average rotational constants given in Table VII.

\section{DISCUSSION}

\section{Use of Spectroscopic and Electron-Diffraction Data for Structure Determination}

As is well known, high-resolution spectroscopy and electron diffraction are powerful and complementary experimental methods for determining gas-phase molecular structure. In the following cases spectroscopy is by far the more suitable method: (a) For diatomic and for some of the simple polyatomic molecules such as linear and bent $\mathrm{XY}_{2}$, where high-resolution spectroscopy is applicable and where precise $r_{e}$ and $r_{z}$ structures can be determined uniquely from the rotational constants of a single isotope species; (b) For molecules such as OCS and $\mathrm{NF}_{3}$ where the equilibrium rotational constants sufficient to determine the complete $r_{a}$ substitution structure can be obtained for various isotope species.

Conversely, for certain nonpolar molecules with large moments of inertia, electron diffraction is the only method available at present for determining precise internuclear distances in gas phase.

In many of the other situations, the use of electron diffraction distances, which may or may not be sufficient to determine the structure uniquely, should be effective to decrease the uncertainty in the spectroscopic average structure, as has been shown for ethane and diborane in the present study. On the other hand, spectroscopic moments of inertia may be helpful to discriminate closely spaced and nonequivalent internuclear distances, if any, and to calibrate the scale factor with more accuracy. Major limitations of the electron-diffraction method may be overcome in this way. ${ }^{12,13}$

\section{Merits of $r_{g}$ and $r_{s}$ Representations}

While the most complete description of "molecular geometry" seems to be the equilibrium internuclear distances $r_{b}$ plus the harmonic and anharmonic potential constants around the equilibrium positions, this description is available for only a limited number of simple molecules. For the rest of the molecules, the average structures $r_{g}$ and $r_{z}$ are probably among the best possible representations of molecular geometry, since both structures have clear physical significance, and since they are more easily accessible from experiment than the $r_{e}$ structure.

When the average lengths of chemical bonds are of primary concern, the $r_{g}$ distance, ${ }^{1}$ which represents "the average value of an instantaneous internuclear distance," should be a more suitable measure than $r_{z}$. On the other hand, $r_{g}$ distances for nonbonded atom pairs do not correspond exactly to any geometrical arrangement consistent with the $\boldsymbol{r}_{\boldsymbol{g}}$ distances for bonded pairs. As is well known in terms of the linear and nonlinear shrinkage effect, ${ }^{35,36}$ effective bond angles calcu-

${ }^{25}$ Y. Morino, J. Nakamura and P. W. Moore, J. Chem. Phys. 36, 1050 (1962).

is Y. Morino, S. J. Cyvin, K. Kuchitsu, and T. Iijima, J. Chem. Phys. 36, 1109 (1962). 
lated from bonded and nonbonded $r_{g}$ distances are, in general, different from average or equilibrium bond angles. In this connection, the $r_{z}$ structure $^{2}$ is more convenient than $r_{\theta}$ as a representation of the geometrical arrangement of atoms, since $r_{z}$ corresponds to "the distance between the average positions of atoms with respect to a molecule-fixed coordinate system." 1-3 However, the $r_{z}$ distance for a bond should be interpreted as the average projection of the bond onto the line joining the equilibrium positions of the atoms in question $\left(r_{e}+\langle\Delta z\rangle\right)$ in the local Cartesian coordinate system of Eq. (1) instead of a real average bond distance $\left(r_{e}+\langle\Delta r\rangle\right)$. In any event, the difference between the $r_{g}$ and $r_{z}$ representations is not a serious problem since they are readily interconvertible within current experimental uncertainty (except for large-amplitude cases).
The $r_{b}$ distances of bonded atom pairs can be estimated approximately ${ }^{4,6,10,11}$ from the corresponding $r_{0}$ distances by the use of a parameter of bond-stretching anharmonicity $a$, which may be transferred from the corresponding diatomic molecules. ${ }^{37}$ On the other hand, there is very limited information at present about the difference between average and equilibrium bond angles. Existing experimental evidence to date, however, suggests that the difference may be only a small fraction of a degree.

\section{ACKNOWLEDGMENTS}

The author wishes to thank Professor Yonezo Morino for his encouragement and Professor L. S. Bartell for his hospitality and valuable advice.

${ }^{87} \mathrm{~K}$. Kuchitsu and Y. Morino, Bull. Chem. Soc. Japan 38, 805 (1965).

THE JOURNAL OF CHEMICAL PHYSICS VOLUME 49, NUMBER 10 15 NOVEMBER 1968

Analysis of Distortion Effects of the $\mathrm{H}_{2} \mathrm{O}$ Molecule

\author{
Dianne Garrett Bruscato \\ University of Louisville, Louisville, Kentucky \\ AND \\ VINCENT A. Gentusa \\ Northeast Louisiana State College, Monroe, Louisiana
}

(Received 26 June 1968)

\begin{abstract}
The method of moments is used to determine the centrifugal-distortion constants of the water molecule. However, to obviate the inclusion of sixth-power terms of the angular-momentum operators in the Hamiltonian, an expression obtained from a second-order transformed Hamiltonian is included to determine the independent centrifugal-distortion constants. By application of the method to infrared spectral data, nine distortion constants were obtained, as were the rotational constants.
\end{abstract}

\section{INTRODUCTION}

The general motion of a molecular system may be considered as the vibrational motion of the individual nuclei plus the rotational motion of the system as a whole. The latter type of motion gives rise to "centrifugal" forces, which cause distortion effects on the individual nuclei. Thus, the Hamiltonian expression describing the motion of the molecule, must include zero-order terms and perturbation terms, arising from the centrifugal effects. These perturbation terms are functions of the total angular-momentum components and certain parameters called centrifugal-distortion constants.

From the method of moments, as developed by Parker and Brown, ${ }^{1-4}$ expressions relating spectral

${ }^{1}$ L. C. Brown and P. M. Parker, J. Chem. Phys. 27, 1108 (1957).

2 P. M. Parker and L. C. Brown, J. Chem. Phys. 30, 909 (1959).

P. M. Parker and L. C. Brown, J. Chem. Phys. 31, 1227 (1959).

'P. M. Parker and L. C. Brown, Am. J. Phys. 27, 509 (1959). data to the parameters of the molecular system may be obtained. Since the water molecule is a very "stretchy" molecule, if $P^{6}$ terms in the Hamiltonian are neglected, these expressions are not sufficient to describe its motion. Hence, we have resorted to a perturbation treatment given by Nielsen ${ }^{5}$ for additional information. With this information we have obtained equations which allow us to calculate the centrifugaldistortion constants from experimental spectral data.

\section{THEORY}

Since the method of moments is the basis for our development, a brief discussion of the method, as given by Parker and Brown, ${ }^{1-4}$ will be given as introduction to our problem.

In matrix notation the energy eigenvalues of the Schrödinger equation

$$
H \psi_{n}=E \psi_{n}
$$

\footnotetext{
${ }^{6}$ H. H. Nielsen, Rev. Mod. Phys. 23, 90 (1951).
} 